\title{
Perception of health and health practices of five Filipino elders
}

\author{
Nena Bonuel* \\ Houston Methodist Hospital, Houston, Texas, United States
}

Received: July 16, 2017

DOI: $10.5430 /$ jnep.v8n5p68
Accepted: November 9, 2017 Online Published: December 20, 2017

URL: https://doi.org/10.5430/jnep.v8n5p68

\begin{abstract}
It is projected that the United States will not have a single racial or ethnic majority by 2055 . Health care providers need to be aware and knowledgeable of other cultures in order to deliver culturally competent care. Equally important is for health care providers to recognize their own biases and to identify and respect differences when delivering care. It is important to know the health and health beliefs of other cultures to effectively provide quality of care. This descriptive exploratory study using fieldwork was focused on the perceptions on health, being healthy, and health practices of 5 Filipino Elders. Elders were asked 7 focused, descriptive questions through interviews about health, being healthy, and health practices in their home to elicit information on the use of traditional remedies, health habits, illness practices, and resources for assistance and health information. Common themes revealed that elders' view of a healthy person is a state of balance between physical and mental well-being. Physical appearances and mobility were part of their perception of health. Elder's perception of an unhealthy person were poor emotional outlook, immobility, and nutritional deficiency.
\end{abstract}

Key Words: Culturally competent care, Filipino elders, Health beliefs, Illness practices, Traditional remedies

\section{INTRODUCTION}

The face of America has been rapidly changing, but never at the pace occurring now. The total population of the white Americans currently the "baseline" at $70 \%$ of the total population will decline to $50 \%$ of the total US patient population by 2060. The African American population will rise from $12.7 \%$ (40.7 million individuals) in 2015 to $14.3 \%$ (50 million African Americans) by 2035. The Latin American population, which is the fastest growing group, will dramatically climb from $17.6 \%$ (56.5 million individuals) in 2015 to $22.3 \%$ (87.5 million) in 2040 . The Asian population currently at $3.6 \%$ (10 million) will increase by 2050 to $9.3 \%$ (37.5 million). Native Hawaiian and Pacific Islanders make up $0.4 \%$ (1.4 million) of the total US population, ${ }^{[1]}$ and there are 2.03 million Arabs living in the United States according to the 2013 Census. ${ }^{[2]}$

The United States is projected to be even more diverse in coming decades. According to the US Census Bureau, ${ }^{[2]}$ in 2014 there were more than 20 million children under 5 years old living in the United States, of which $50.2 \%$ were minorities. The United States will not have a single racial or ethnic majority by the year 2055 as a result of immigration. At least 59 million immigrants have arrived in the United States in the past 50 years. Nowadays, a near-record $14 \%$ of the country's population is foreign-born compared with just $5 \%$ in 1965 . The majority of US population growth is projected to be linked to new Asian and Hispanic immigration over the next 5 years. ${ }^{[3]}$ American attitudes about immigration and diversity are for the most part supportive of these

*Correspondence: Nena Bonuel; Email: nbonuel@ houstonmethodist.org; Address: Houston Methodist Hospital, Houston, Texas, United States. 
changes. More Americans say immigrants strengthen the country than say they burden it, and most say the increasing ethnic diversity of the United States makes it a better place to live.

Despite the notable progress of the national initiative in Healthy People 2020, disparities persist in illness and death among racial and ethnic groups in comparison to the whole US population. ${ }^{[4,5]}$ Non-Hispanic African Americans are at least 50\% more likely than non-Hispanic whites to die prematurely from heart disease or stroke. ${ }^{[6]}$ Asian/Pacific Islander adults are $60 \%$ more likely to have acute hepatitis $\mathrm{B}$, a major cause of liver disease, than non-Hispanic whites. ${ }^{[7]}$ African American, Native Hawaiian/other Pacific Islander, and Hispanic adults all have rates of HIV infection diagnosis that range from 3 to 9 times the rate of non-Hispanic whites. ${ }^{[6]}$ Hispanics, non-Hispanic blacks, and those of mixed race all have higher rates of diabetes than non-Hispanic whites. ${ }^{[6]}$ The mortality rates for non-Hispanic black infants and American Indian/Alaska Native infants are far higher than those of non-Hispanic white infants. ${ }^{[6]}$ African Americans are more likely than non-Hispanic whites to be hospitalized for heart failure, and the same goes for Native Hawaiians and other Pacific Islanders. Asian Americans are less likely than whites to receive recommended hospital care for pneumonia. ${ }^{[5]}$

US Filipinos according to existing population-based study in California have high burden of chronic conditions. They have the highest adjusted rates with overweight condition among all other racial/ethnic group in California. ${ }^{[8]}$ Between 1992-2011, obesity prevalence tripled for Filipinos based on the National Health Interview survey. ${ }^{[9]}$ They also carry disproportionately burden for diabetes and hypertension. ${ }^{[10]}$ Because Filipinos have significantly higher risk for adverse metabolic syndrome due to the BMI range, a call for Asianspecific BMI cutoff points is needed to define overweight, obesity, and metabolic risk screening. ${ }^{[11]}$ The purpose of this descriptive study was to determine the perceptions of health, being healthy, and health practices of Filipino elders.

\subsection{Purpose of the study}

To determine the perceptions of health, being healthy, and health practices of Filipino elders.

\subsection{Review of the literature}

Filipinos are very resilient people. Often they are compared to bamboo trees that bend when the wind blows and go straight back up again when the wind stops. There is certain "apartness", according to Patterson ${ }^{[12]}$ that "no matter how accurately the blows of fate seem aimed at him, there is always something that remain unhit, as though part of him were permanently elsewhere" (p. 35). Filipinos have the ability to take on circumstances as they come without any complaint and with a sense of humor. They love to laugh and can always find something amusing even in the most trying situations. ${ }^{[13]}$ Friendliness, sweetness, generosity, and hospitality are part of the predominant demeanors of the people. ${ }^{[12]}$

It was in the 20th century that male Filipinos arrived in the United States as contract laborers to do agricultural work. Most of them were students who studied and supported themselves at the same time to complete their education. Other Filipinos were also actively recruited in Hawaii and in the valleys of California. Most of the Filipinos moved to California after 2 major strikes in Hawaii. In California these men were viewed as outcasts, and most of them remained unmarried because the ratio of men to women was $14: 1$. Filipinos who dated white women were persecuted, which resulted in many anti-Filipino riots in California. A judge handed down a ruling in the 1930s that classified Filipinos as a member of the Mongolian race, making mixed marriages in California invalid. The education levels as well as English skills of these Filipino elders were low. These Filipino elders experienced hardship and were the survivors of the earlier immigration era. By the mid-1980s, there were 50,000 to 60,000 older Filipinos in the United States, and men outnumbered women 4 to 1 . When the quota was abolished in 1965, a second surge of migration from the Philippines was composed of predominantly young professionals. ${ }^{[14-16]}$ These young professionals were predominantly nurses sponsored by American recruiters to alleviate the nursing shortage in the United States. Once they established themselves in the United States, these nurses sponsored their families to join them. It is typical for the wife to work in the United States and the husband and children to remain in the Philippines. Thus, while pursuing the goal for a better life in the United States, separation, distance, and fragmentation of Filipino families are common. ${ }^{[17]}$ Despite these barriers, the Filipino family remains intact due to their strong belief in God and the influence of Catholicism, which is the predominant religion of the Filipino people, who consider divorce a sin.

The Filipinos are the second largest Asian group in the United States, according to the 2013 US Census, and they are 3.4 million strong. There are at least 6,862,490 Filipino Americans who belong to the subgroup of age 65 , a slightly smaller number than Chinese American elders. The Filipino average per capita income is below the national average. Typically, $75 \%$ of Filipino households' income is pooled from 3 or more working adults. The Filipino household is usually an extended family wherein the grandparents stay with their married children. It is also common to see grandparents babysitting their grandchildren. ${ }^{[18,19]}$ 
In the 1990s, 90\% of Filipino Americans aged 65 and over were born outside the United States and $57 \%$ were naturalized. Also, 89\% spoke a language other than English, $56 \%$ claimed they did not speak English very well, and $17 \%$ were classified as being linguistically isolated. Close to half $(43 \%)$ had less than a 9 th grade education; $16 \%$ had undergraduate or advanced degrees, $18 \%$ were still employed, $8 \%$ lived in poverty, and $30 \%$ of those 75 years and older lived alone. ${ }^{[19]}$ In 2008, Filipino Americans aged 65 years and older accounted for $9.9 \%$ of the total Filipino American population and an estimated $35.4 \%$ were disabled. The median household income was $\$ 74,983$, which is $25 \%$ more than the national average. The poverty rate of Filipino Americans aged 65 and older is $7.7 \%$, lower than that of the total US geriatric population (9.5\%) and other Asian Americans. Many Filipino American older adults can communicate in English but prefer to speak their native languages when ill or under stress. ${ }^{[18]}$

In the Philippines, Filipino or Tagalog is the national language and English is the second language. Approximately 80 to 100 ethnic languages are spoken in this 7,000-island archipelago. At least 8 major regional languages are spoken: Pilipino/Tagalog (29.6\%), Cebuano (24.2\%), Ilocan0 (10.3\%), Ilongo (9.2\%), Bicolano (3.5\%), Waray (4\%), Kapampangan $(2.8 \%)$, and Pangasinanes $(<1 \%)$, as cited in McBride. ${ }^{[19]}$ Among Filipino Americans, "Tag-lish," a hybrid language that is a combination of English and Tagalog, is spoken by many and is widely used in the Filipino American media, visual and performing arts, and marketing campaigns. Two-thirds of Pilipino households speak a non-English language.

\subsection{Conceptual framework}

The author used the cultural assessment framework (CAF) model developed by Huff and Kline. ${ }^{[20]}$ The CAF provides the health care professional a foundation with which to better understand the similarities and differences between the mainstream culture and the specific ethnic population through an assessment approach. The CAF integrates 5 levels of assessment that are essential when planning an intervention for any multicultural group. The 5 major levels of assessment for the $\mathrm{CAF}$ are as follows:

(1) Cultural or ethnic group-specific demographic characteristics.

(2) Cultural or ethnic group-specific epidemiological and environmental influences.

(3) General and specific cultural or ethnic group characteristics.

(4) General and specific health care beliefs and practices.

(5) Western health care organization and service delivery variables.

The author chose level 4, which is focused on the health behavior practices assessment area. The model explains that major differences exist between unique patient populations and the mainstream Western population in perceptions of health and illness. Furthermore, the model claims that there are differences of opinion concerning how to approach treatment, compliance with prescribed treatments, and participation in health promotion initiatives. The other area to explore is the response to illness and diseases. Many multicultural groups initially seek help from their own traditional healer or use traditional medicines that are obtained from the community, handed down through the family, or recommended by close friends of the family. These traditional health practices can be challenging when combined with Western medical treatments. The role of the health care provider, therefore, is to determine how to blend the traditional medicine with the Western medical model that would be beneficial and accepted by the targeted population. ${ }^{[20]}$ The health care provider must also recognize that food preferences, environmental factors like sanitation, habits, and educational-behavioral practices influence individuals' perceptions of health and illness. The most important, according to Hakim and Wegman, ${ }^{[21]}$ is the "client's perception of health, since this perception drives the client's response to care" (p. 162).

\section{Methods}

\subsection{Design}

This descriptive study used fieldwork to explore the perception of health and health practices of Filipino Elders.

\subsection{Sample and setting}

The study used a convenient sample of 5 Filipino elders living in southwestern Houston. All the Filipino elders lived close to each other. The ages of the participants ranged from 66 to 76 years, all were female, and all lived with their children who were nurses. Three participants were widowed and 2 were married. The husband of one of the married elders resided in the Philippines. The 5 participants originated from different parts of the Philippines. The author visited each of the Filipino elders in the morning in their children's home during one weekend. To gain rapport, the author offered to take the elders' blood pressure; the elders were very excited to have their blood pressure taken. Consent was explained by the author and signed by the elders who were reassured of participant confidentiality.

\subsection{Instrument}

To understand the health and health practices of the Filipino elders, a content validated instrument used 7 focused, de- 
scriptive questions. The interview questions were designed to engage the participants in discussion of the following:

(1) The Filipino elders' perception of health.

(2) Health maintenance and illness practices.

(3) Health practices of ancestors.
Additional questions were used to determine the elders' use of traditional remedies, health habits, illness practices, and resources for assistance and health information (see Figure $1)$.

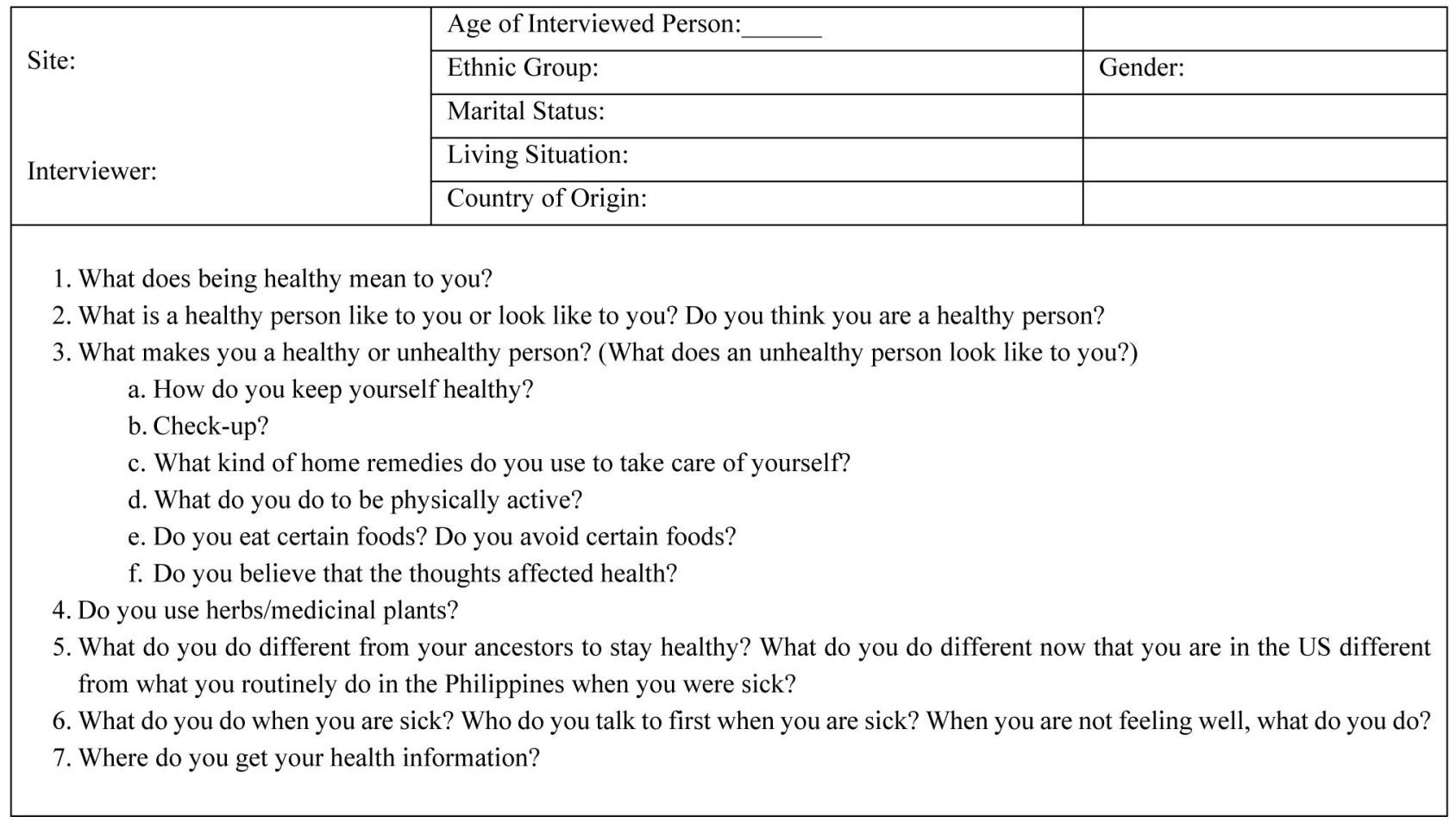

Figure 1. Instrument used to interview Filipino elders

\subsection{Data collection}

Basic oral interviewing techniques were used to question the elders about their perspectives on health and health practices. The questions were used to engage the Filipino elders to initiate a conversation and stimulate discussion about their health practices to maintain health. The elders were also asked about the health practices of their ancestors. The author attempted to record key words, phrases, and sentences in response to the questions. The author conducted an analysis, immersed herself in the data, and identified common threads and recurring themes.

\section{ANALYSis AND Discussion}

The study was exploratory and descriptive in nature using qualitative approaches. Qualitative approaches deal with the issue of human complexity, the ability of humans to shape and create self-experiences, and the idea that truth is a composite of realities. The study relied heavily on understanding the human experience as it is lived. Qualitative analysis was used at the end of the day, and the collected responses were analyzed for themes regarding the participants' perceptions of health, health maintenance, and health practices. Common themes of health beliefs and practices among the Filipino elders were identified and documented.

\subsection{Perception of being healthy}

When the Filipino elders were asked what being healthy meant to them, the following responses were obtained: "maganda ang katawan" (beautiful body), "not fat or thin," "happy but not depressed," "you can have a good body but if you are depressed, you are not healthy," "not sick," "active," "Mabuti ang katawan"(good body), "beautiful," "active," and "satisfied in life." Their concept of a healthy person seemed to include the physical as well as the mental well being of an individual. This concept is supported by Chin and Bigby, ${ }^{[22]}$ who indicated that health is viewed as holistic and life energies need to be balanced through linkage of mind, body, and soul.

\subsection{Characteristics of a healthy person}

The participants were asked what a healthy person looks like, and their responses seemed to rely heavily on physical ap- 
pearances and their mobility, as exhibited in their responses, such as: "beautiful," "face don't look like a problem," "active," "dieting, same weight," and "looks good." When asked whether they were healthy, 2 of the elders responded positively and the other 3 said they suffered from arthritis, hemorrhoids, and diabetes. The one who answered positively further elaborated by saying that she is active in the social circle. On the other hand, one who answered negatively informed the interviewer that she went to the Philippines and ate the wrong foods and made her diabetes worse. The latter is supported by the explanatory model of the theory of illness that lies in naturalistic causes as presented in the study by McBride. ${ }^{[19]}$ In this theory, illness is attributed to nature from things like the weather, stressful situations, food, and medications that do not agree with the body. This concept is supported by information gathered from the Management Sciences for Health and Office of Minority Health and Bureau of Primary Health Care's Provider's Guide to Quality and Culture, ${ }^{[23]}$ which states that diseases are caused by a sudden change in the environment. For example, rheumatism and respiratory disease are caused by high winds and rainy weather.

\subsection{Description of a person that is not healthy}

The participants were asked what an unhealthy person looks like, and 3 common themes were identified: poor emotional outlook, immobility, and nutritional deficiency by way of responses like "they look sad and pitiful," "weak, cannot walk," "does not work," "always in bed," and "cannot eat and no appetite." Physical activity in the form of exercise, walking around the neighborhood, dancing, stretching, biking, using a treadmill, skipping rope, housework, and mowing the lawn were the predominant answers by the participants when asked how to keep oneself healthy. All 5 elders were friends, and walking around the neighborhood was one of their routines for maintaining health and fostering camaraderie. The second answer concerned prevention of disease by having a regular checkup with a physician and the use of medication for specific illness such as arthritis, constipation, stomach pain, indigestion, and headache. Foods that were associated with keeping one healthy, according to the elders, were eating a lot of seafood, chicken, and vegetables and avoiding dog and cat meat. Eating in moderation was also mentioned as a way to maintain health. The participants also believed that mental outlook influenced the health of the individual. One elder believed that if you have a problem and think too much, it will make you sick, and another elder recommended just relaxing, taking it easy, and avoiding worries.

According to McBride, ${ }^{[19]}$ Filipinos believe that health and illness are considered to be on the same equilibrium with the physical and mental well being with 3 explanatory models being mystical, personalistic, or natural causes. There is a belief that the soul gets out of the body during sleep when someone has a nightmare ("bangungot") after a heavy meal, which causes a person to die. This belief is associated with the mystical causes. A person's illness may be caused by a witch ("inkanto") or sorcerer ("mankukulam") and may be viewed as a punishment. A healer or a priest who is considered stronger in spirit may be able to heal the individual and counteract the bad spirits. Use of holy oils and wearing religious objects or amulets ("anting anting") is common. ${ }^{[18,19]}$ Filipinos believe that evil spirits can cause an individual to be sick. As reported in Traditional Filipino Health Practices, ${ }^{\text {[21] }}$ the evil spirit ("manga ga mud") may cast a spell on a disliked person by obtaining personal items such as a piece of cloth, a piece of hair, pictures, or a piece of jewelry. Food poisoning is also believed to be the doings of the evil spirits. To undo the spell, the individual needs to see a herbalaryo (witch doctor). The treatment involves a ritual sprinkling of rice, dining, and dancing of unmarried individuals to the sound of Pilipino music. The conclusion of the ceremony involves saying a prayer to the cursed individual, which is claimed to eliminate the curse of that person.

\subsection{On the use of herbs and medicinal plants}

The interesting part of the whole interview was when the participants were asked if they were using medicinal herbs. The predominant answer was the use of boiled guava leaves for cleaning the intestines; the leaves are also used to treat constipation, hemorrhoids, and indigestion and for washing minor cuts. There was also a reference to pito-pito, meaning 7 leaves, which consists of suha (citrus leaves), banana, mitla, mango, guava, avocado, and duhat (black berry). Pito-pito, according to the elders, is good for cleaning the urinary track and the intestines and also decreases blood pressure. The participants drank these leaves like tea during lunchtime. A study by Ndubani et al. ${ }^{[24]}$ to investigate how far cultural constructs can determine seeking treatment for diarrhea in the poorer population of Lusaka, Zambia, revealed that people seek a source from multiple venues. The data collection methods in that study included discussions, a household survey, a survey of practitioners of traditional medicine, and interviews with locals who were attending an urban health center with persistent diarrhea. Of the 1,743 respondents, $1.5 \%$ indicated that they seek help from a herbalist and $0.7 \%$ self-medicate with home remedies from herbs. Guava leaves were mentioned by 7 respondents, but the survey mentioned that there may have been some underreporting out of embarrassment or fear of compromising the relationship with the biomedical workers in the health center. Guava leaves as a medicinal plant were also mentioned by Hadwiger and Had- 
wiger $^{[25]}$ in an article on the transcultural nursing experience in the Philippines wherein 8 senior students and 1 faculty member traveled to the Philippines to gain knowledge of the health care system and the country's culture. Healthy Philippines 2,000 was a national program wherein there was an emphasis on a "Clean and Green" campaign that addressed how to use medicinal plants.

Also mentioned was the use of drinking 2 teaspoonful of vinegar to lower cholesterol and blood pressure. Vinegar and a little salt in warm water were used for a sponge bath to lower fever. There was numerous mention of the use of tea: green tea, Lipton tea, Zendo tea, and Gen-mai-cha tea to aid in digestion and to neutralize fatty foods. One participant mentioned the use of corn hair for urinary tract infection and to clean the kidney. Another elder claimed the use of coconut water to be good to prevent kidney stones. Yet another elder claimed the use of Pandan (palm) leaves to be good for the kidney.

\subsection{Habits different from ancestors to stay healthy and current health habits different from habits back home when sick}

When the participants were asked the difference between their ancestors' way of staying healthy and their own, the common response was that their parents were farmers and were active in the fields. When asked what they did differently now that they were in United States from what they routinely did in the Philippines, most of the respondents reported that they now used medication over-the-counter when they have minor aches and pains. There was also mention of drinking hot water when not feeling well.

According to the cohort studies of McBride, ${ }^{[19]}$ one of the Filipino indigenous health beliefs is the principle of balance (Timbang), wherein one believes that rapid shifts from "hot" to "cold" lead to illness and a warm environment is essential to maintain optimum health. The humoral balance of the body is affected by sudden changes in the weather, such as a cool breeze in the evening hours that lowers temperature, the presence of the hot sun right after a lengthy rain, and vapor rising from the soil, which upset the body's balance simply by blowing on the body surface. This humoral concept is reinforced by the information taken from the Management Sciences for Health ${ }^{[23]}$ regarding the principle of balance (Timbang), which recommends a balanced diet of "hot" and "cold" foods. Excessive intake of hot food was perceived to result in arthritis and hypertension, whereas cold foods might cause cancer and anemia.

One elder mentioned that she had malaria for 3 months and she treated herself with ground guava leaves, vinegar, and Published by Sciedu Press salt, wrapped in banana leaves and cooked in charcoal. She then squeezed the juice and rubbed it on her entire body. She claimed that her malaria was resolved by the herbal concoction. She further explained that in the United States, her left arm went numb and she went to the hospital after 3 days. She had a CAB, stayed in the hospital for 4 days, and went home with pain relievers. She was proud to add that she exercises regularly, takes a bath daily, and eats food in moderation. The treatment with guava leaves for malaria is substantiated by a study conducted by Okrah et al. ${ }^{[26]}$ in center de Recherche en Sante de Nouna, Burkina Faso, where in they boiled and made concoctions out of guava leaves. This liquid can be taken as a drink or can be used for a sponge bath or rubbed in the skin, depending on the acuity of illness.

\subsection{Action taken when sick and who to turn for help}

When asked to whom they talk first when they are sick, 4 elders responded that first they will talk to their children who are nurses. One responded that she talked to her friend. McBride ${ }^{[19]}$ wrote that Filipino elders develop some form of a care path when sick. Initially, they try to treat themselves, depending on the severity of symptoms. They may selfmedicate with food, herbs, or over-the-counter medicines. They may discuss symptoms with their friend or a person they trust and respect, such as someone from the church or a family member. Seeking medical attention is dependent on their experience from social interactions in the community, the previous experience of other family members, and the experience of the elders themselves when seeking medical care. McBride noted that for specific symptoms, elders seek the assistance of either a friend or a family member who is a health care professional. Filipinos who have chronic disease believe that compliance with prescribed treatments is directly proportional to control of the disease.

\subsection{Source of health information}

When asked where they got their health information, 2 of the respondents said they got it from their children. One of them said that she gets it from a medical doctor. Two interesting answers were that one gets her information in the Parade Magazine from the Houston Chronicle and another answered that she gets her information from Nurse's Week.

\subsection{Limitations of the study}

It is important and beneficial to discuss limitations of the study. For the elders to open up and willingly engage in participating in the study, the consent and the 7 focused questions should have been written in Tagalog, the Filipino language. Although the 5 elders understood and spoke English, on numerous occasions they requested the author to repeat the questions. Three elders were skeptical of answer- 
ing the questions for fear of how the information would be used. It would also help to give prospective participants the consent and the questionnaire beforehand so that discussions with the children, who were all working as nurses, about participating in the study could take place and allay the anxiety of the elders regarding answering questions. One elder had to call her daughter and inform her of the purpose of the visit although 3 days before the actual fieldwork, the author was introduced to the daughter and informed the daughter personally of the nonexperimental study. It would also be difficult to generalize perceptions of health and health practices of the 5 elders because the sample were all female and they were parents of children who are in the health care profession. Thus, accessibility may not be a major problem. There were also some time constraints in doing the fieldwork that would have prepared the elders about the study. Word of mouth was used to disseminate information regarding participation in the study to a colleague who was a nurse, and the nurse's colleague's mother supplied the sample population. It would be helpful to have variable samples rather than a homogeneous sample to gain better insight on the perceptions of health and health practices of Filipino elders.

\section{CONClusions, SUMMARY, AND RECOM- MENDATION}

The overall health indicators for Asian American and $\mathrm{Pa}$ cific Islanders consider this population to be the healthiest in the United States. Although they are considered the healthiest, there is a paucity of available demographic and health data specific to Filipinos because they are under the general heading of Asian American and Pacific Islanders. There is a need to aggregate the data to determine variations in income, education, and health, which may hide some serious socioeconomic and health problems within the ethnic groups.

The general theme that the Filipino elders believe regarding health is that it is a state of balance between the physical and mental well-being of the individual. Any sort of physical activity such as walking, stretching, and dancing is important to be incorporated in the daily routine to be healthy. To have a good outlook in life is equally important to be healthy.
There is a predominant use of medicinal plants such as guava leaves and other tea leaves as a liquid concoction and in baths that needs to be further explored in a larger sample size. Use of medicinal plants is not uncommon in this particular ethnic group, and medicinal plant usage is also predominant in other cultural groups.

It is important for the health care provider, to be aware and be knowledgeable of other cultures in order to deliver culturally competent care. Policy makers should continue the dialogue on equality with regards to access to care and reducing care disparity. Nursing education should continue on highlighting culturally congruent care by developing innovative ways of teaching cultural competency. More research is needed to highlight the importance of culture and its impact to the health of the individual, their participation to plan of care and to their overall well-being. Equally important is for all of us; who provides care to the patient; is our knowledge of personal self-bias and attitude, and one must be able to identify and respect differences when delivering caring services. Each patient is entitled to safe, quality, individualized care in order to obtain effective treatment outcomes. Nurses are morally and ethically obligated to care for their patients with respect and dignity regardless of differences in race, socioeconomic standing, sexual orientation, religion, ability, and disease conditions. It is not only the nurse's obligation but also the nurse's duty to ensure that equality and justice to health care access is provided to all patients.

The changing demographics and economics of our growing multicultural world, and the long-standing disparities in the health status of people from culturally diverse backgrounds, have challenged us and organizations to consider cultural diversity a priority. These changing demographics accentuate the importance of cultural diversity in a profession where the patient-provider relationship is key to determining the quality of care. As health care providers, it is important that we understand the values and beliefs of the patients we serve to be effective in providing culturally sensitive care.

\section{CONFlicts of InTERest Disclosure}

The author declares that there is no conflict of interest.

\section{REFERENCES}

[1] Office of Minority Health. Profile: Hispanic/Latino Americans [Internet]. Washington (DC): US Department of Health and Human Services [cited 2017 May 1]. Available from: https : //www . mino rityhealth.hhs.gov/omh/browse. aspx?lvl=3\&lvlid=64

[2] US Bureau of Census [Internet]. Washington (DC): US Department of Commerce. Available from: https://www. census.gov/ [cited
2017 May]

[3] Cohn D, Caumont A. 10 Demographic trends that are shaping the U.S. and the world [Internet]. Washington (DC): Pew Research Center [published March 31, 2016; cited 2016 Mar 31]. Available from: http://www. pewresearch.org/fact-tank/2016/03/ 31/10-demographic-trends-that-are-shaping-the-u-s -and-the-world/ 
[4] Healthy People 2020. Disparities [Internet]. Washington (DC): US Department of Health and Human Services [cited 2017 May 1]. Available from: https://www.healthypeople.gov/2020/abo ut/foundation-health-measures/Disparities

[5] Agency for Healthcare Research and Quality. 2012 National Healthcare Disparities Report [Internet]. Washington (DC): US Department of Health and Human Services [cited 2017 Jun 22]. Available from: http://archive.ahrq.gov/research/findings /nhqrdr/nhdr12/index.html

[6] Centers for Disease Control and Prevention. CDC Health Disparities and Inequalities Report-United States, 2013 [Internet]. Atlanta (GA): Centers for Disease Control and Prevention [published November 22, 2013; cited 2013 Nov 22]. Available from: https ://www.cdc.gov/mmwr/pdf/other/su6203.pdf

[7] US Department of Health and Human Services, Office of Minority Health. Hepatitis and Asian Americans. Washington (DC): US Department of Health and Human Services [cited 2017 Jun 7]. Available from: http://minorityhealth.hhs.gov/omh/brows e. $\operatorname{aspx} ? \operatorname{IvI}=4 \& \operatorname{Ivlid}=50$

[8] Jih J, Mukherjea A, Vitinghoff E, et al. Using appropriate body mass index cut points for overweight and obesity among Asian American. Preventive Medicine [published April 13, 2014; cited Sept. 2017]. Available from: https://www.ncbi.nlm.nih.gov/pmc/artic les/PMC4217157/

[9] Singh G, Lim S. Dramatic increases in obesity and overweight prevalence among Asian subgroups in the United States [published September 19, 2013; cited Sept. 2017]. Available from: https: //www . hindawi.com/journals/isrn/2013/898691/

[10] Karter A, Schillinger D, Adams A, et al. Elevated rates of Diabetes in Pacific Islanders and Asian subgroups. The Diabetes Study of Northern California (DISTANCE). Diabetes care [published August 13, 2012; cited Sept. 2017]. Available from: http://care.diabe tesjournals.org/content/diacare/36/3/574.full.pdf

[11] Hsu W, Araneta M, Kanaya A, et al. BMI cut points to identify at -risk Asian American for type 2 diabetes screening. Diabetes care [published January 2015, cited Sept. 2017]. Available from: https://www.ncbi.nlm.nih.gov/pmc/articles/PMC4 392932/pdf/150.pdf

[12] Patterson JH. History tells the story. In: The Philippines, A Journey Through the Archipelago. New York: Archipelago Press; 1997; 35-45 p.

[13] Perez RD. Oh, what joys where'er you look. In: The Philippines, A Journey Through the Archipelago. New York: Archipelago Press; 1997.

[14] McNamara Keith, Batalova J. Filipino Migrants in the United States [Internet]. Washington (DC): Migration Policy Institute [published July 21, 2015; cited 2017 Jun 30]. Available from: http://www.migrationpolicy.org/article/filipi no-immigrants-united-states

[15] Pellicer M. Presented at: Annual Meeting of the Organization of Chinese-Americans. Available from: http://www.atl-filam.o rg/Pellicer_Metty/Our_Aged.htm

[16] Dela Cruz M, Agbayani-Siewart. Filipino Americans [Internet]. In: Asian-Nation: The Landscape of Asian America. Asian Nation [published 2003; cited 2017 Jun 30]. Available from: http: //www . as ian-nation.org/filipino.shtml\#sthash.xYnVT1Cj.dpbs

[17] Globalization 101. The case of the Filipino Nurses [Internet]. State University of New York: The Levin Institute [published 2017; cited 2017 Jul 1]. Available from: http: //www.globalization101.o rg/the-case-of-the-philippine-nurses/

[18] Dela Cruz M, Periyakoil VJ. Health and Health Care of Filipino American Older Adults [Internet]. [published 2010; cited 2017 Jul 1]. Available from: https://sgec.stanford.edu/

[19] McBride H. Health and health care of Filipino American Elders. Stanford (CA): Stanford University School of Medicine [published 2002; cited 2004 Jun 16]. Available from: http: //www . stanford .edu/group/ethnoger/filipino.html

[20] Huff R, Kline M. Promoting Health in Multicultural Populations. Thousand Oaks (CA): Sage; 1999.

[21] Hakim H, Wegman D. A comparative evaluation of the perception of health of elders of different multicultural backgrounds. J Community Health Nurs. 2002; 19(3): 161-171. https ://doi.org/10.1207/ S15327655JCHN1903_04

[22] Chin JL, Bigby J. Care of Asian Americans. Journal of Cross-cultural Medicine. Philadelphia (PA): American College of Physicians; 2003.

[23] Management Sciences for Health and Office of Minority Health and Bureau of Primary Care. A provider's guide to quality and culture [seminar]. Medford (MA): Management Sciences for Health. Available from: https://www.msh.org/resources/provider s-guide-to-quality-culture

[24] Ndubani P, Kelly P, Farthing MJG, et al. Local understanding of adult diarrheal disease and its treatment in an area of high HIV-seroprevalence in Zambia. Trop Med Int Health. 1998; 3(10): 783-787. https://doi.org/10.1046/j.1365-3156.19 98.00303.x

[25] Hadwiger M, Hadwiger S. Transcultural nursing experience in the Philippines. Nurse Educ. 1999; 24(1): 12-15. https ://doi.org/ 10.1097/00006223-199901000-00005

[26] Okrah J, Traore C, Pale A, et al. Community factors associated with malaria prevention by mosquito nets: an exploratory study in rural Burkina Faso. Trop Med Int Health. 2002; 7(3): 240-248. https://doi.org/10.1046/j.1365-3156.2002.00856.x 